\title{
Fluorescence Spectroscopy and Molecular Docking Approach to Probe the Interaction between Dehydroeburicoic Acid and Human Serum Albumin
}

\author{
Shijie Zheng1, Shude Yang², Xianhao Cheng², Tolgor Bau, Yu Li', Rui Zhang², Haiying Bao1* \\ ${ }^{1}$ Engineering Research Center, Chinese Ministry of Education for Edible and Medicinal Fungi, Jilin Agricultural University, \\ Changchun, China \\ ${ }^{2}$ Shandong Key Lab of Edible Mushroom Technology, Ludong University, Yantai, China \\ Email: ‘baohaiying2008@126.com,2755@sdu.edu.cn
}

How to cite this paper: Zheng, S.J., Yang, S.D., Cheng, X.H., Bau, T., Li, Y., Zhang, R. and Bao, H.Y. (2019) Fluorescence Spectroscopy and Molecular Docking Approach to Probe the Interaction between Dehydroeburicoic Acid and Human Serum Albumin. Advances in Microbiology, 9, 21-37.

https://doi.org/10.4236/aim.2019.91003

Received: December 12, 2018

Accepted: January 11, 2019

Published: January 14, 2019

Copyright $\odot 2019$ by author(s) and Scientific Research Publishing Inc. This work is licensed under the Creative Commons Attribution International License (CC BY 4.0).

http://creativecommons.org/licenses/by/4.0/

\begin{abstract}
The interaction between dehydroeburicoic acid (DeEA), a triterpene purified from medicinal fungi and the major transport protein, human serum albumin (HSA), were systematically studied by fluorescence spectroscopy, synchronous fluorescence spectroscopy, three-dimensional fluorescence spectroscopy and molecular docking approach under simulated physiological conditions. The intrinsic fluorescence of HSA was quenched through the combination of static and dynamic quenching mechanism. DeEA cannot be stored and carried by HSA in the body at higher temperature. The hydrogen bonding, hydrophobic force and van der Waals force were major acting forces. The site II was the major binding site. The energy transfer could occur with high probability and the binding distance was $3.29 \mathrm{~nm}$. The binding process slightly changed the conformation and microenvironment of HSA. The DeEA molecule entered the hydrophobic cleft of HSA and formed the hydrogen bonding with Glu-492 and Lys-545.
\end{abstract}

\section{Keywords}

Human Serum Albumin, Dehydroeburicoic Acid, Interaction, Fluorescence, Molecular Docking

\section{Introduction}

Dehydroeburicoic acid (DeEA) is a triterpene purified from medicinal fungi such as Taiwanofungus camphoratus, Fomitopsis officinalis, Fomitopsis pinicola 
and Wolfiporia cocos [1] [2]. The molecular structure of DeEA was presented in Figure 1. It can protect the liver from $\mathrm{CCl}_{4}$-induced oxidative stress and tissue injuries [3]. It possesses analgesic and anti-inflammatory effects [4]. It exhibits antitumor activity against the human glioblastoma U87MG [2] [5]. It has an excellent therapeutic potential for the treatment of type 1 diabetes and type 2 diabetes [6] [7]. Human serum albumin (HSA) is significant in the blood circulatory system. It contains 585 amino acid residues [8] [9]. It is stabilized by 17 disulfide bridges [10]. It consists of three homologous domains (domain I-III). And each domain contains two subdomains (IA, IB, IIA, IIB, IIIA and IIIB). It has two high-affinity binding sites (site I and site II) [11] [12]. The site I is located in the subdomain IIA. The site II is located in the subdomain IIIA. The interaction between biologically active substances (drugs or natural products) and HSA affects the bioavailability, distribution, free state concentration and metabolism of biologically active compounds in the bloodstream [13] [14]. In addition, the interaction between biologically active compounds and HSA changes the structure and function of HSA [15]. Therefore, probing the interaction between DeEA with HSA is significant to deeply understand the pharmacodynamics and pharmacokinetics properties of DeEA.

In summary, we characterized the interaction between DeEA and HSA by fluorescence spectroscopy, synchronous fluorescence spectroscopy, three-dimensional fluorescence spectroscopy and molecular docking approach. The quenching mechanism, the binding constant, the number of binding site, the thermodynamic parameters and the acting force were estimated according to the fluorescence spectroscopy data. Synchronous fluorescence spectroscopy and threedimensional fluorescence spectroscopy were performed to probe the conformational and microenvironmental changes of HSA during the binding process. Molecular docking was performed to show the interaction between DeEA

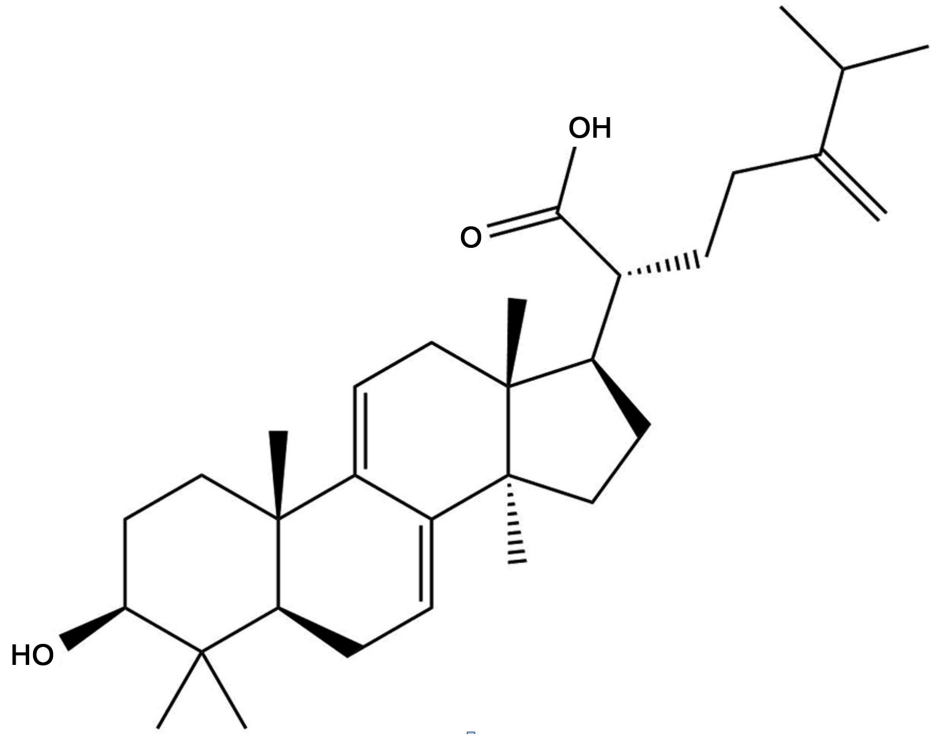

Figure 1. The molecular structure of dehydroeburicoic acid. 
and amino acid residues of HSA.

\section{Materials and Methods}

\subsection{Reagents and Materials}

HSA (A1887; fatty acid and globulin free) was purchased from Sigma-Aldrich. DeEA was purchased from Aikon Biopharmaceutical R\&D Co. Ltd. (Jiangsu, China). Site probes (Warfarin and Ibuprofen) were purchased from Shanghai Xiyuan biological technology Co. Ltd. (Shanghai, China). The concentration of HSA stock solution is $1.0 \times 10^{-4} \mathrm{M}$ in $1.0 \times 10^{-2} \mathrm{M}$ phosphate buffer solution ( $\mathrm{pH}$ 7.40). The concentration of DeEA stock solution is $1.0 \times 10^{-4} \mathrm{M}$. The concentration of site probes stock solution is $1.0 \times 10^{-4} \mathrm{M}$. All reagents used are of analytical grade. The water used for the experiment was ultrapure water. Stock solutions were prepared weekly and stored in the dark at $4^{\circ} \mathrm{C}$.

\subsection{Fluorescence Spectroscopy Measurements}

Fluorescence spectroscopy measurements were performed on the Thermo Scientific Lumina fluorescence spectrophotometer. The excitation wavelength was $280 \mathrm{~nm}$. The emission wavelength was recorded from 285 to $500 \mathrm{~nm}$. The fixed concentration of $3.0 \mu \mathrm{M}$ HSA $(3.5 \mathrm{~mL})$ was continuously titrated with the addition of DeEA. The photomultiplier tube (PMT) voltage, the excitation slit, the emission slit, the response time, the integration time and the scan speed were set at $500 \mathrm{~V}, 2.5 \mathrm{~nm}, 2.5 \mathrm{~nm}, 1.0 \mathrm{~s}, 20 \mathrm{~ms}$ and $1200 \mathrm{~nm} / \mathrm{min}$, respectively. The experiments were performed at 288,298 and $308 \mathrm{~K}$.

\subsection{Synchronous Fluorescence Spectroscopy}

Synchronous fluorescence spectroscopy measurements were performed on the Thermo Scientific Lumina fluorescence spectrophotometer. Synchronous fluorescence spectroscopy was recorded from 200 to $500 \mathrm{~nm}$. The scanning interval between the excitation and emission wavelength $(\Delta \lambda)$ was set at 15 and $60 \mathrm{~nm}$, respectively. Other parameters are the same as the fluorescence spectroscopy measurements. The experiments were performed at $298 \mathrm{~K}$.

\subsection{Three-Dimensional Fluorescence Spectroscopy}

Three-dimensional fluorescence spectroscopy measurements were performed on the Thermo Scientific Lumina fluorescence spectrophotometer. The excitation wavelength ranged from 200 to $360 \mathrm{~nm}$. The emission wavelength ranged from 200 to $660 \mathrm{~nm}$. Other parameters are the same as the fluorescence spectroscopy measurements. The experiments were performed at $298 \mathrm{~K}$.

\subsection{Site Marker Competitive Experiments}

The site marker competitive experiments were performed on the Thermo Scientific Lumina fluorescence spectrophotometer. Warfarin is marker for site I. Ibuprofen is marker for site II [12] [16]. The warfarin-HSA system was continuous- 
ly titrated with the addition of DeEA. The ibuprofen-HSA system was continuously titrated with the addition of DeEA. Other parameters are the same as the fluorescence spectroscopy measurements. The experiments were performed at $298 \mathrm{~K}$.

\subsection{UV-Visible Absorption Spectroscopy Experiments}

The UV-visible absorption spectroscopy experiments were performed on the Shimadzu UV-2550 spectrophotometer. The UV-visible absorption spectroscopy was recorded from 200 to $500 \mathrm{~nm}$. The experiments were performed at room temperature.

\subsection{Molecular Docking}

Molecular docking was performed using the AutoDock [17]. The 3D structure of DeEA was optimized using Gaussian 09 before docking simulations [18]. The binding site was defined using one grid of $120 \times 120 \times 120$ points at the subdomain IIIA [19]. Lamarckian genetic algorithm was used with a total of 100 runs. The DeEA-HSA complex was visualized and analyzed using Chimera [20].

\section{Results and Discussion}

\subsection{Quenching Mechanism}

Fluorescence spectroscopy was performed to probe quenching mechanism of the interaction between DeEA and HSA. The fluorescence spectroscopy of HSA in the absence and presence of DeEA were presented in Figure 2. The fluorescence intensity of HSA obviously decreased with increasing the concentration of

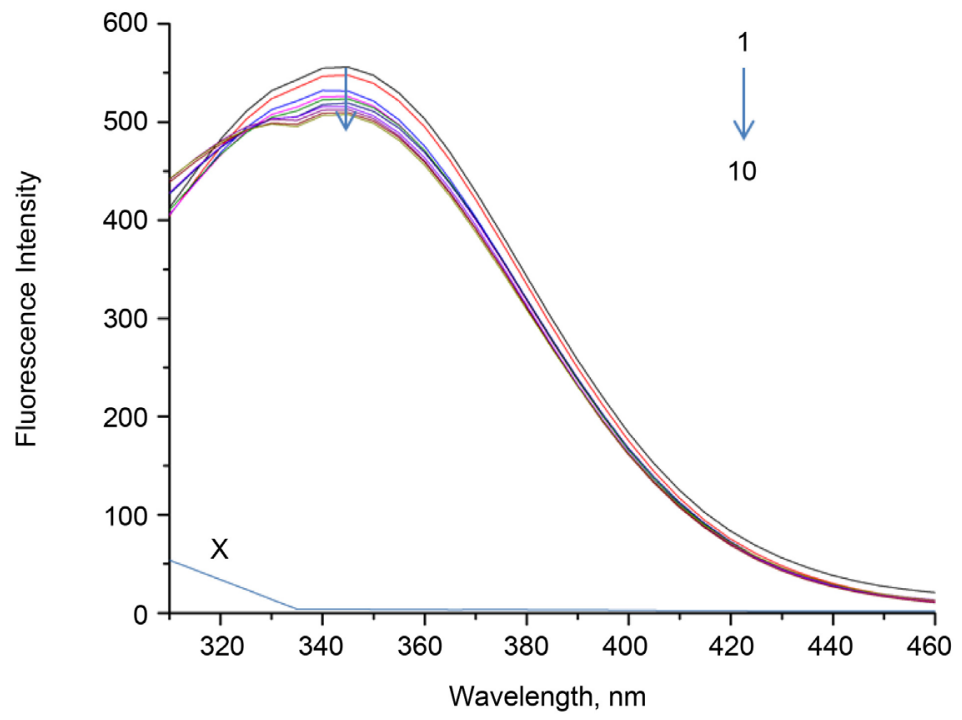

Figure 2. The effect of DeEA on the fluorescence of HSA, $c($ HSA $)=3.0$ $\mu \mathrm{M}$. Lines 1 - 10: $c($ DeEA $)=0,0.143,0.286,0.429,0.572,0.715,0.858$, $1.001,1.144,1.287 \mu \mathrm{M}$, respectively; $\lambda_{e x}=280 \mathrm{~nm} ; \lambda_{e m}=345 \mathrm{~nm} ; \mathrm{pH}=7.4 ; T=$ $298 \mathrm{~K}$. Curve $\mathrm{x}$ shows the emission spectrum of DeEA only, $c($ DeEA $)=$ $0.143 \mu \mathrm{M}$. 
DeEA. However, the fluorescence intensity of DeEA was almost equal to 0. In general, for dynamic quenching, the quenching constant increases with increasing the temperature [13]. For static quenching, the quenching constant decreases with increasing the temperature [13]. The Stern-Volmer equation was used to estimate the possible quenching mechanism [21] [22].

$$
\frac{F_{0}}{F}=1+K_{q} \tau_{0}[Q]=1+K_{s v}[Q]
$$

where $F_{0}$ is the fluorescence intensity of free HSA, $F$ is the fluorescence intensity of the HSA-DeEA system, $K_{S V}$ is the Stern-Volmer quenching constant, $[Q]$ is the concentration of DeEA, $K_{q}$ is the biomolecule quenching rate constant, $\tau_{0}$ is the lifetime of the fluorophore in the absence of DeEA and the fluorescence lifetime of the biopolymer is $10^{-8} \mathrm{~s}$ [23].

The Stern-Volmer plots for fluorescence quenching of the DeEA-HSA system at different temperatures were presented in Figure 3. The $K_{S V}$ and $K_{q}$ value were collected in Table 1 . The value of $K_{S V}$ obviously increased with increasing the temperature, which indicated that the quenching mechanism was dynamic quenching [24]. However, the value of $K_{q}$ was much greater than the maximum scatter collision quenching constant $\left(2.0 \times 10^{10} \mathrm{M}^{-1} \cdot \mathrm{S}^{-1}\right)$, which showed that the quenching mechanism was static quenching [25]. The results demonstrated that DeEA quenched the fluorescence of HSA through the combination of static and dynamic quenching mechanism.

\subsection{Binding Constant and Binding Site}

For the binding process, the binding constant $\left(K_{A}\right)$ and the number of binding sites $(n)$ were estimated according to the following equation [26].

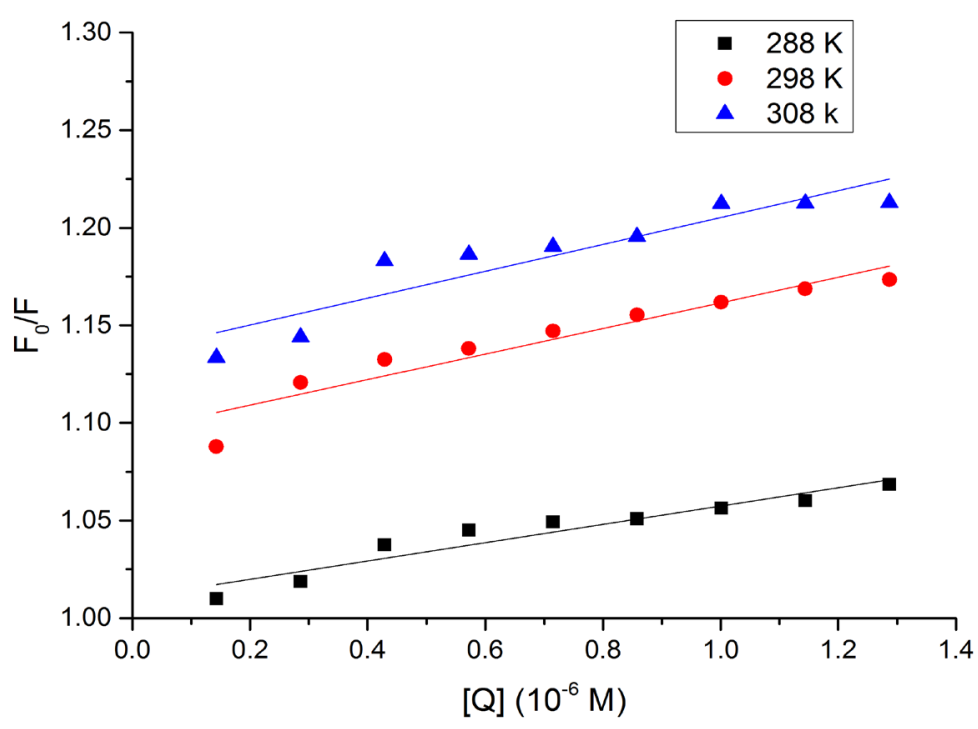

Figure 3. The Stern-Volmer plots for fluorescence quenching of the DeEA-HSA system at different temperatures, $c($ HSA $)=3.0 \mu \mathrm{M} ; c$ $($ DeEA $)=0.143-1.287 \mu \mathrm{M} ; \lambda_{e x}=280 \mathrm{~nm} ; \lambda_{e m}=345 \mathrm{~nm} ; \mathrm{pH}=7.4 ; T=$ 288, 298 and $308 \mathrm{~K}$. 
Table 1. The biomolecule quenching rate constant and the Stern-Volmer quenching constant for the DeEA-HSA system at different temperatures.

\begin{tabular}{cccc}
\hline$T(K)$ & $K_{q}\left(\mathrm{~L} \cdot \mathrm{mol}^{-1} \cdot \mathrm{s}^{-1}\right)$ & $K_{S V}\left(\mathrm{~L} \cdot \mathrm{mol}^{-1}\right)$ & $R$ \\
\hline 288 & $4.690 \times 10^{12}$ & $4.690 \times 10^{4}$ & 0.9588 \\
298 & $6.558 \times 10^{12}$ & $6.558 \times 10^{4}$ & 0.9545 \\
308 & $6.879 \times 10^{12}$ & $6.879 \times 10^{4}$ & 0.9282 \\
\hline & $\log \left(\frac{F_{0}-F}{F}\right)=\log K_{A}+n \log [Q]$ &
\end{tabular}

where the meanings of $F_{0}, F$ and $[Q]$ are the same as for Equation (1). The double-logarithm plots for fluorescence quenching of the DeEA-HSA system at different temperatures were presented in Figure 4 . The $K_{A}$ and $n$ value were collected in Table 2 . The $K_{A}$ value obviously decreased with increasing the temperature, which revealed that the stability of the DeEA-HSA complex was reduced during the binding process [27] [28] [29]. Moreover, the number of binding sites approximated to 1 at $288 \mathrm{~K}$, which suggested that there was almost one site for the interaction between DeEA and HSA at this temperature. And the $n$ value obviously decreased with increasing the temperature, which showed that DeEA could not bind to HSA at higher temperature. Since the stability of the DeEA-HSA complex significantly reduced and the value of $n$ was much less than 1 with increasing the temperature during the binding process. Therefore, DeEA could bind to HSA at $288 \mathrm{~K}$. However, DeEA could not be stored and carried by HSA in the body at higher temperature [30].

\subsection{Thermodynamic Parameters and Acting Forces}

In order to estimate the acting forces, the thermodynamic parameters enthalpy change $(\Delta H)$, entropy change $(\Delta S)$ and free energy change $(\Delta G)$ of the binding process were calculated according to the Van't Hoff equation [31] [32].

$$
\begin{aligned}
\ln K_{A} & =-\frac{\Delta H}{R T}+\frac{\Delta S}{R} \\
\Delta G & =\Delta H-T \Delta S
\end{aligned}
$$

where $K_{A}$ is the binding constant at the corresponding temperature, $R$ is the molar gas constant and $T$ is the thermodynamic temperature.

The thermodynamic parameters were collected in Table 3 . The negative value of $\Delta G$ indicated that the binding process was spontaneous. The negative value of $\Delta H$ revealed that the binding process was exothermic. According to the theory of Ross and Subramanian, we concluded that the hydrogen bonding and the van der Waals force were major acting forces for the interaction between DeEA and HSA [31]. Therefore, the hydrogen bonding and the van der Waals force were major acting forces. And the binding process were spontaneous and exothermic. 


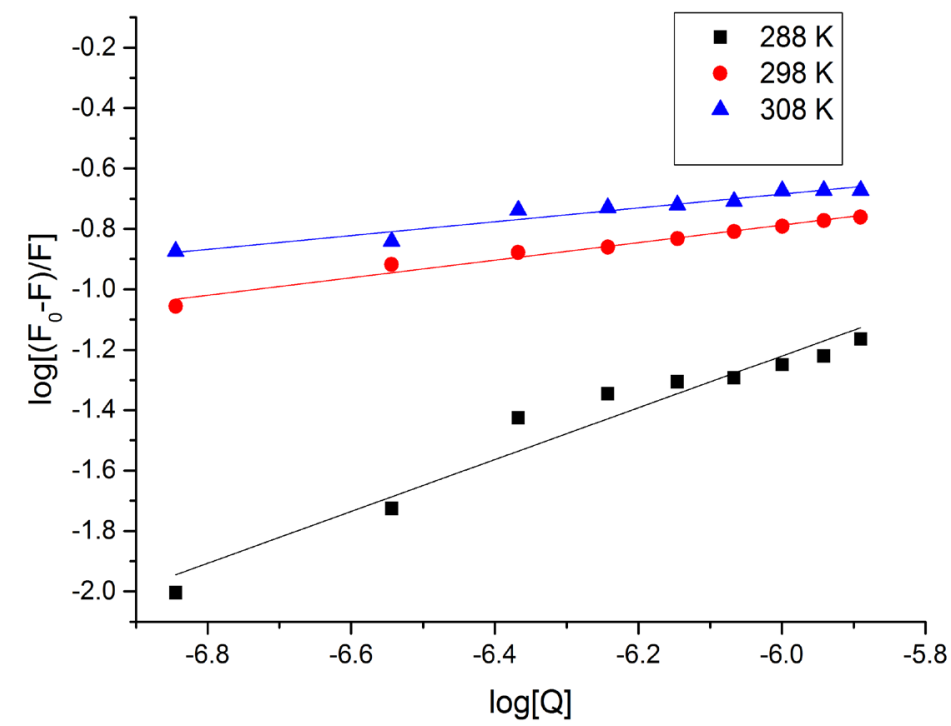

Figure 4. The double-logarithm plots plot for fluorescence quenching of the DeEA-HSA system at different temperatures, $c$ (HSA) $=3.0 \mu \mathrm{M}$; $c($ DeEA $)=0.143-1.287 \mu \mathrm{M}$, respectively; $\lambda_{e x}=280 \mathrm{~nm} ; \lambda_{e m}=345 \mathrm{~nm}$; $\mathrm{pH}=7.4 ; T=288,298$ and $308 \mathrm{~K}$.

Table 2. The binding constant and the number of binding site for the DeEA-HSA system.

\begin{tabular}{cccc}
\hline$T(K)$ & $n$ & $K_{A}\left(\mathrm{~L} \cdot \mathrm{mol}^{-1}\right)$ & $R$ \\
\hline 288 & 0.858 & 8407.948 & 0.9745 \\
298 & 0.290 & 9.022 & 0.9879 \\
308 & 0.229 & 4.867 & 0.9706 \\
\hline
\end{tabular}

Table 3. The thermodynamic parameters for the interaction between DeEA and HSA.

\begin{tabular}{ccccc}
\hline$T(K)$ & $K_{A}\left(\mathrm{~L} \cdot \mathrm{mol}^{-1}\right)$ & $\Delta G\left(\mathrm{~kJ} \cdot \mathrm{mol}^{-1}\right)$ & $\Delta H\left(\mathrm{~kJ} \cdot \mathrm{mol}^{-1}\right)$ & $\Delta S\left(\mathrm{~J} \cdot \mathrm{mol}^{-1} \cdot \mathrm{K}^{-1}\right)$ \\
\hline 288 & 8407.948 & -19.337 & -277.340 & -895.847 \\
298 & 9.021 & -10.378 & & \\
308 & 4.867 & -1.420 & & \\
\hline
\end{tabular}

\subsection{Site Marker Competitive Experiments}

The site marker competitive experiments were performed to locate the major binding site for the interaction between DeEA and HSA. HSA has two principal sites located in hydrophobic pocket. The sites are located in the hydrophobic cavities in subdomains IIA and IIIA, which exhibit similar chemical properties [33]. The binding cavities associated with subdomains IIA and IIIA are also referred to as site I and site II. Warfarin is marker for the site I. Ibuprofen is marker for the site II [34] [35]. The warfarin-HSA system and ibuprofen-HSA system were continuously titrated with the addition of DeEA, respectively. Then, the experiment data were analyzed by Equation (2). The binding constant of the DeEA-HSA system before and after addition of site markers were presented in 
Table 4. The binding constant obviously decreased with the addition of ibuprofen, which revealed that warfarin displaced DeEA from the binding site. Therefore, we concluded that site II might be the major binding site for the interaction between DeEA and HSA.

\subsection{Fluorescence Resonance Energy Transfer and Binding Distance}

There was obvious overlap between the fluorescence spectrum of HSA and the absorption spectrum of DeEA in Figure 5. According to the fluorescence resonance energy transfer theory, the binding distance $(r)$ between HSA (donor) to DeEA (acceptor) was calculated [36]. The efficiency of energy transfer $(E)$ depends on $r$ and the extent of spectral overlap ( $)$. The value of $E$ can be calculated by the following equation:

$$
E=1-\frac{F}{F_{0}}=\frac{R_{0}^{6}}{R_{0}^{6}+r^{6}}
$$

where $F_{0}$ is the fluorescence intensity of free HSA, $F$ is the fluorescence intensity of the HSA-DeEA system, $R_{0}$ is the critical energy transfer distance when $E=50 \%$ and $r$ is the binding distance between DeEA and HSA. The value of $R_{0}$ can be calculated according to the Forster's equation.

Table 4. The binding constant of the DeEA-HSA system before and after addition of site probes (warfarin and ibuprofen).

\begin{tabular}{cc}
\hline System & $K_{A}\left(\mathrm{~L} \cdot \mathrm{mol}^{-1}\right)$ \\
\hline HSA + DeEA & 9.022 \\
HSA + DeEA + warfarin & 3.784 \\
HSA + DeEA + ibuprofen & 3.428
\end{tabular}

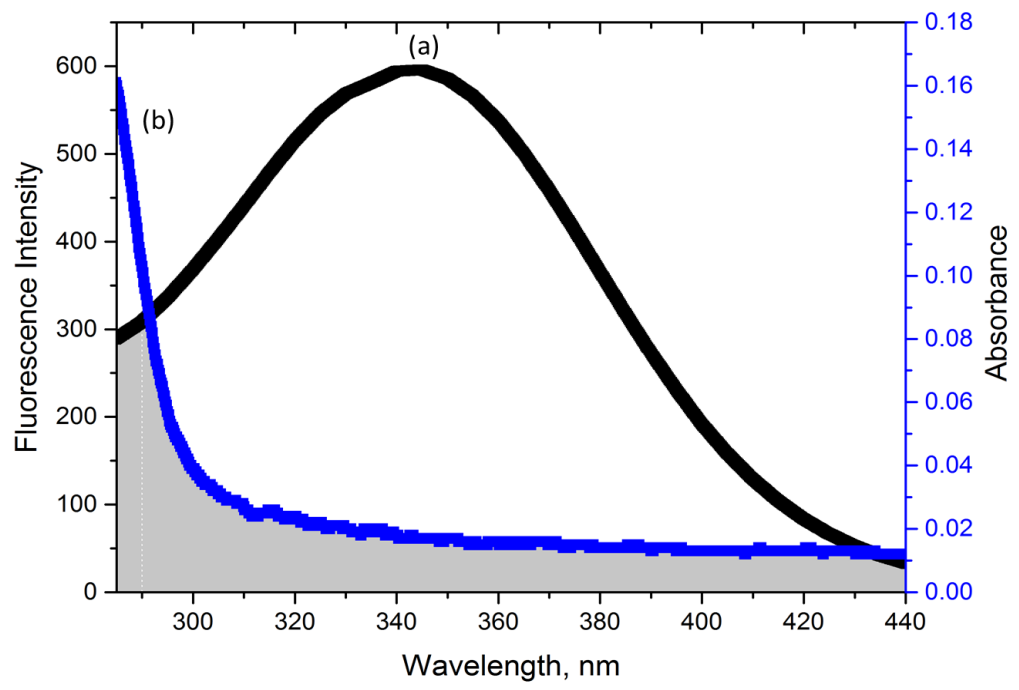

Figure 5. The overlap between the fluorescence spectrum of HSA (a) and the absorption spectrum of DeEA (b), $\lambda_{e x}=280 \mathrm{~nm} ; \lambda_{e m}=345 \mathrm{~nm} ; \mathrm{pH}=$ 7.4; $c($ HSA $)=c($ DeEA $)=3.0 \mu \mathrm{M}$. 


$$
R_{0}^{6}=8.8 \times 10^{-25} k^{2} N^{-4} \Phi J
$$

where $k^{2}$ is spatial orientation factor of the dipole, $N$ is refractive index of the medium and $\Phi$ is the fluorescence quantum yield of HSA. In the present case, $k^{2}=2 / 3, N=1.336$ and $\Phi=0.118$ [37] [38]. The value of $J$ can be calculated by the following equation:

$$
J=\frac{\sum F(\lambda) \varepsilon(\lambda) \lambda^{4} \Delta \lambda}{\sum F(\lambda) \Delta \lambda}
$$

where $F(\lambda)$ is the fluorescence intensity of HSA at wavelength $\lambda$ and $\varepsilon(\lambda)$ is the molar absorption coefficient of DeEA at wavelength $\lambda$.

In view of the above, we calculated that $E=0.15, R_{0}=2.45 \mathrm{~nm}$ and $r=$ $3.29 \mathrm{~nm}, r<7 \mathrm{~nm}$. The value of $R_{0}$ and $r$ were on the $2-8 \mathrm{~nm}$ scale and $0.5 R_{0}<r<1.5 R_{0}$, which indicated that DeEA could interact with HSA [39]. The results revealed that DeEA could interact with HSA and the energy transfer could occur with high probability.

\subsection{Conformational Investigation}

\subsubsection{Synchronous Fluorescence Spectroscopy}

Synchronous fluorescence spectroscopy was performed to probe the conformational and microenvironmental changes of HSA during the binding process. The effect of DeEA on the synchronous fluorescence of HSA was presented in Figure 6. When $\Delta \lambda=15 \mathrm{~nm}$, the shift of $\lambda_{\max }$ was minor from 300.6 to $301.4 \mathrm{~nm}$, which showed that the change of microenvironment around tyrosine residues were trivial [40] [41]. When $\Delta \lambda=60 \mathrm{~nm}$, the shift of $\lambda_{\max }$ remained unchanged, which indicated that the microenvironment around tryptophan residues remained the same [40]. However, the stronger fluorescence quenching of tyrosine residues than tryptophan residues was observed with increasing the concentration of DeEA, which revealed that the binding site was nearer tyrosine residues. The tyrosine residues were mainly located in the subdomain IIIA. Therefore, we concluded that the major binding site was located in the subdomain IIIA (site II). These results were consistent with the site marker competitive experiments.

\subsubsection{Three-Dimensional Fluorescence Spectroscopy}

Three-dimensional fluorescence spectroscopy was performed to explore the conformational and microenvironmental changes of HSA during the binding process. Three-dimensional fluorescence spectroscopy of free HSA and the DeEA-HSA system were presented in Figure 7. The corresponding parameters were collected in Table 5. Peak a $\left(\lambda_{e x}=\lambda_{e m}\right)$ is the Rayleigh scattering peak [42]. Peak b $\left(2 \lambda_{e x}=\lambda_{e m}\right)$ is the second-order scattering peak [42]. Peak $1\left(\lambda_{e x}=280 \mathrm{~nm}\right.$ and $\lambda_{e m}=331 \mathrm{~nm}$ ) mainly exhibits the spectrum characteristic of tyrosine and tryptophan residues, which can reflect conformational changes of HSA [19] [43]. In this study, the fluorescence intensity of peak a and peak b obviously decreased with the addition of DeEA due to the formation of the DeEA-HSA complex. The fluorescence intensity of peak 1 increased with the addition of DeEA, which 

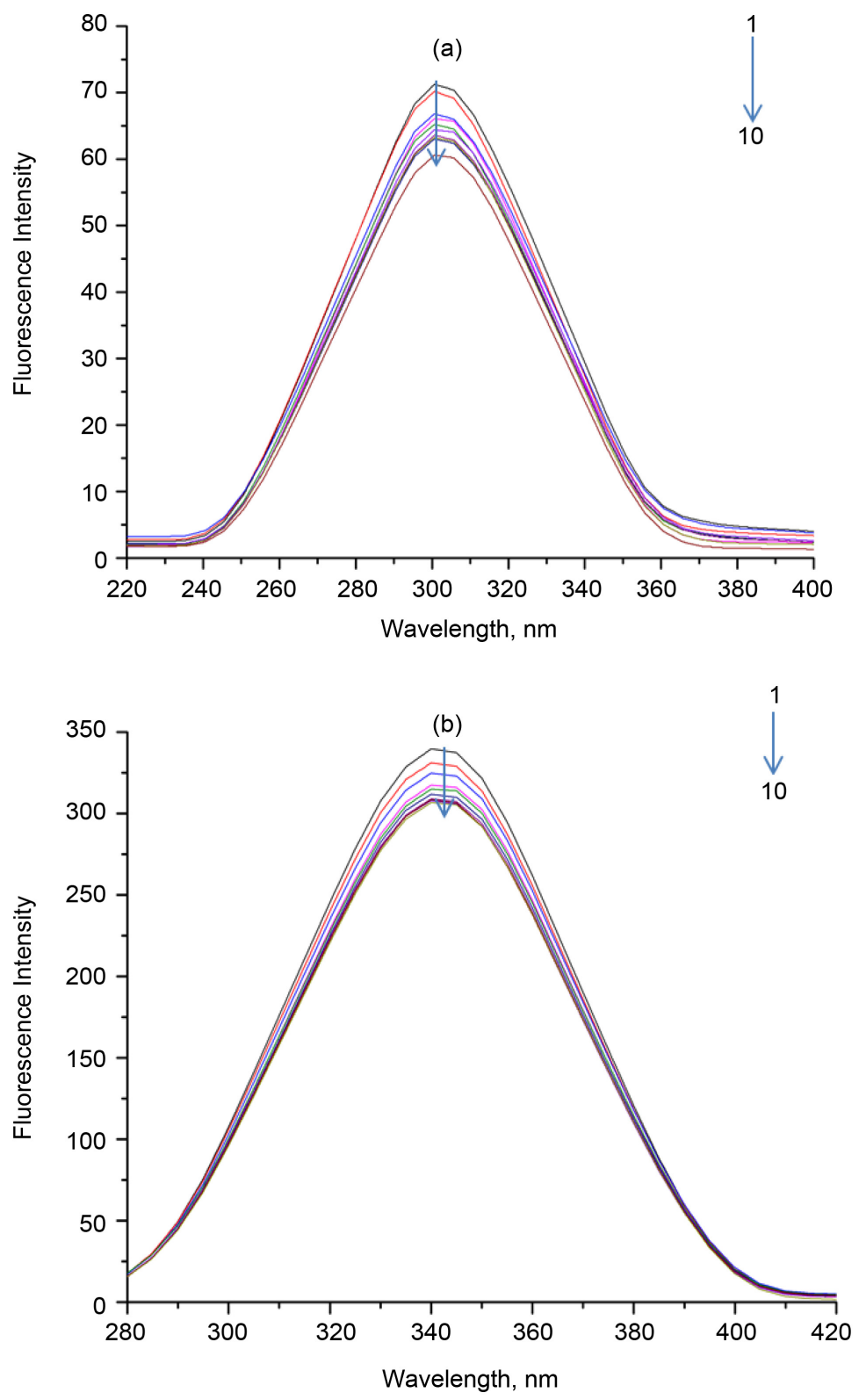

Figure 6. The effect of DeEA on the synchronous fluorescence of HSA: (a) $\Delta \lambda=15 \mathrm{~nm}$; (b) $\Delta \lambda=60 \mathrm{~nm}$. $c($ HSA $)=3.0 \mu \mathrm{M}$. Lines $1-10: c($ DeEA $)=$ $0,0.143,0.286,0.429,0.572,0.715,0.858,1.001,1.144,1.287 \mu \mathrm{M}$, respectively; $\mathrm{pH}=7.4 ; T=298 \mathrm{~K}$.

revealed that the hydrophobic microenvironment near tyrosine residues has been changed [44]. The results showed that DeEA have interacted with HSA and slightly changed the conformation and microenvironment of HSA.

\subsection{Molecular Docking}

In order to show the interaction between DeEA with amino acid residues of HSA, molecular docking was performed. The binding mode of the DeEA-HSA system was presented in Figure 8. The results revealed that the DeEA molecular 


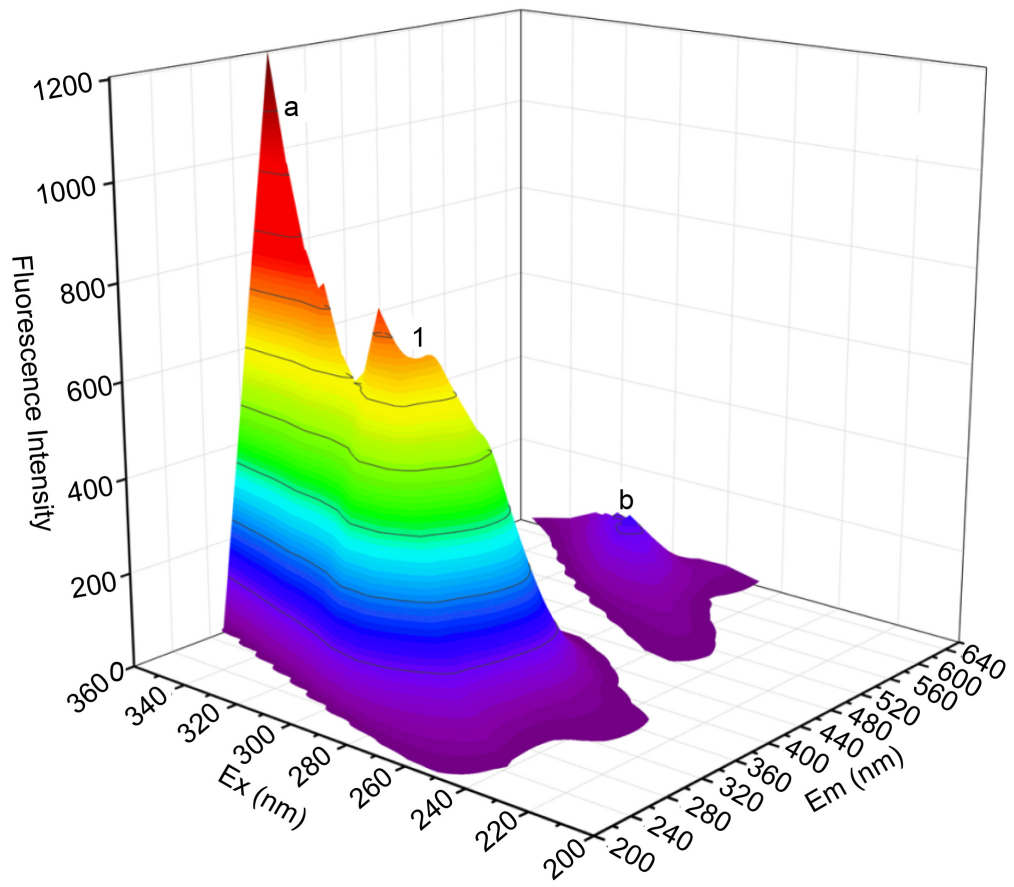

(A)

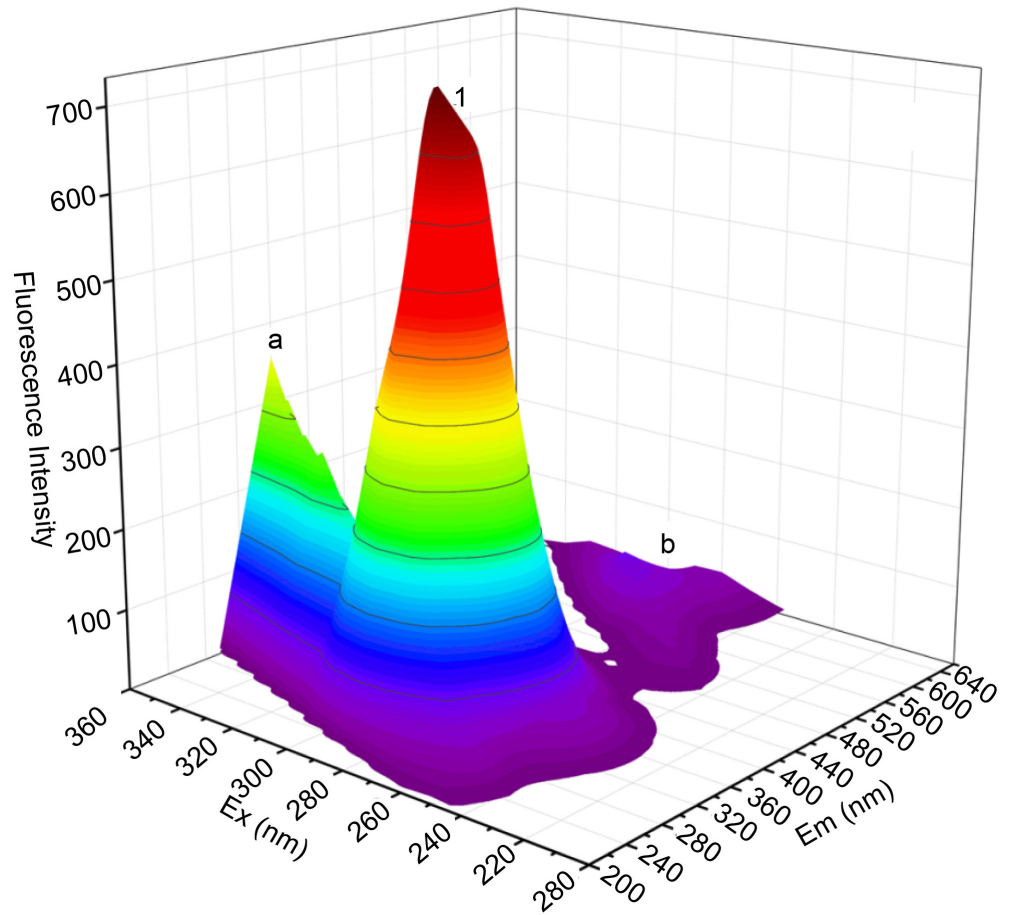

(B)

Figure 7. The three-dimensional fluorescence of free HSA (A) and the DeEA-HSA system (B); $\mathrm{pH}=7.4 ; T=298 \mathrm{~K}$.

located in the cavity surrounded by the following amino acid residues: Asn-405, Val-409, Arg-410, Lys-413, Lys-414, Glu-492, Val-493, Leu-529, Thr-540, Leu-544, Lys-545 and Met-548. The Glu-492 formed hydrogen bonding with the hydrogen atom of the hydroxyl group of DeEA at the distance of $2.571 \AA$. In 


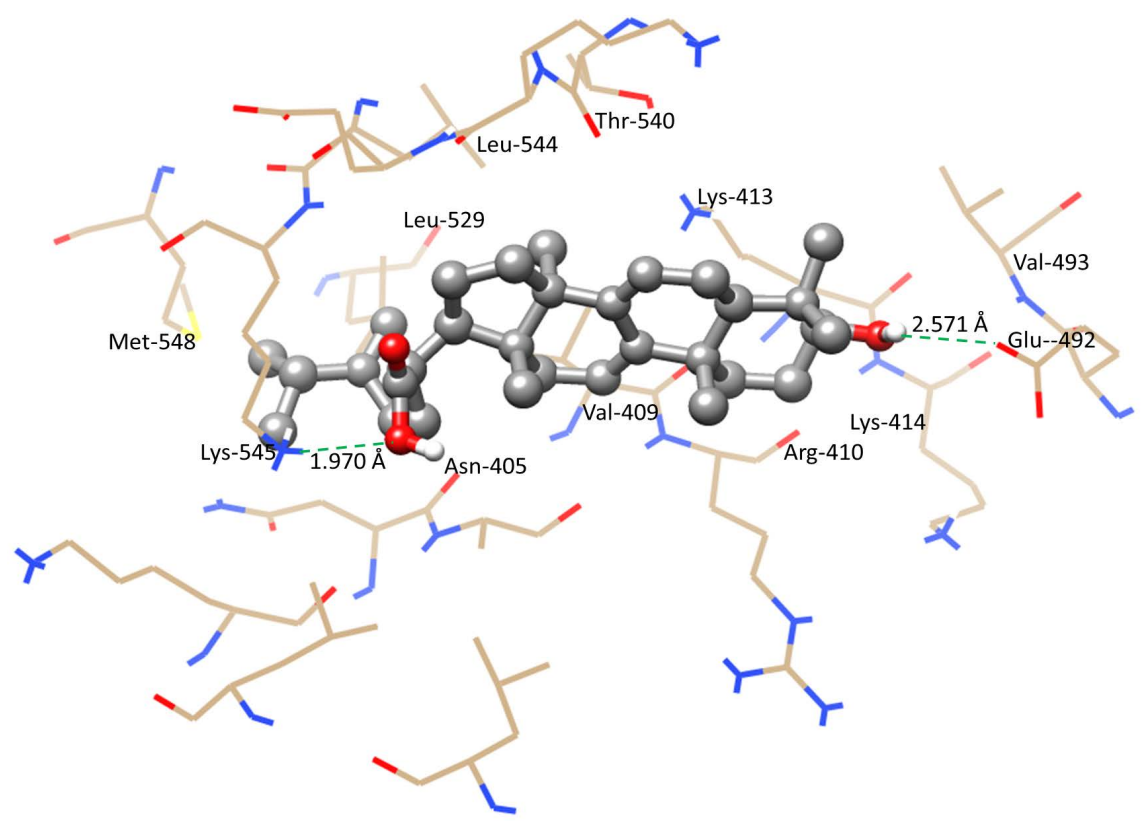

Figure 8. The binding mode of the binding DeEA to HSA, the secondary structure of the protein is shown and the important neighboring amino acid residues are labeled. The ligand structure is represented in the ball-and-stick model and hydrogen bond is indicated by a green dashed line.

Table 5. The three-dimensional fluorescence spectral characteristic parameters of free HSA and the DeEA-HSA system.

\begin{tabular}{|c|c|c|c|}
\hline System ${ }^{\star}$ & Peak & Peak Position $\lambda_{e x} / \lambda_{e m}(\mathrm{~nm} / \mathrm{nm})$ & Intensity \\
\hline \multirow[t]{3}{*}{ Free HSA } & $\mathrm{a}$ & $240 / 240 \rightarrow 360 / 360$ & $13.9 \rightarrow 1185.9$ \\
\hline & $\mathrm{b}$ & $300 / 600$ & 153 \\
\hline & 1 & $280 / 331$ & 557.7 \\
\hline DeEA-HSA & a & $240 / 240 \rightarrow 360 / 360$ & $10.5 \rightarrow 350.6$ \\
\hline \multirow[t]{2}{*}{$(\mathrm{DeEA}=1.001 \mu \mathrm{m})$} & $\mathrm{b}$ & $300 / 600$ & 66.3 \\
\hline & 1 & $280 / 331$ & 672.9 \\
\hline
\end{tabular}

*The concentration of HSA was $3.0 \mu \mathrm{M}$ for each system.

addition, the Lys-545 formed the hydrogen bonding with the oxygen atom of the hydroxyl group of DeEA at the distance of $1.970 \AA$. Hydrophobicity surface of amino acid residues within $5 \AA$ around DeEA was presented in Figure 9. As color changes from blue to red, surface alters from hydrophilic to hydrophobic gradually. The hydroxyl groups of DeEA were in hydrophilic surface and the hydrophobic portion was in hydrophobic surface, which decided the reasonable interaction between DeEA and HSA. The molecular docking results revealed that the hydrogen bonding and hydrophobic force were significant for the interaction between DeEA and HSA, which were consistent with the thermodynamic analysis. The hydroxyl groups of DeEA were important groups during the binding process. The Lys-545 and Glu-492 were important amino acid residues for the interaction between DeEA and HSA. 


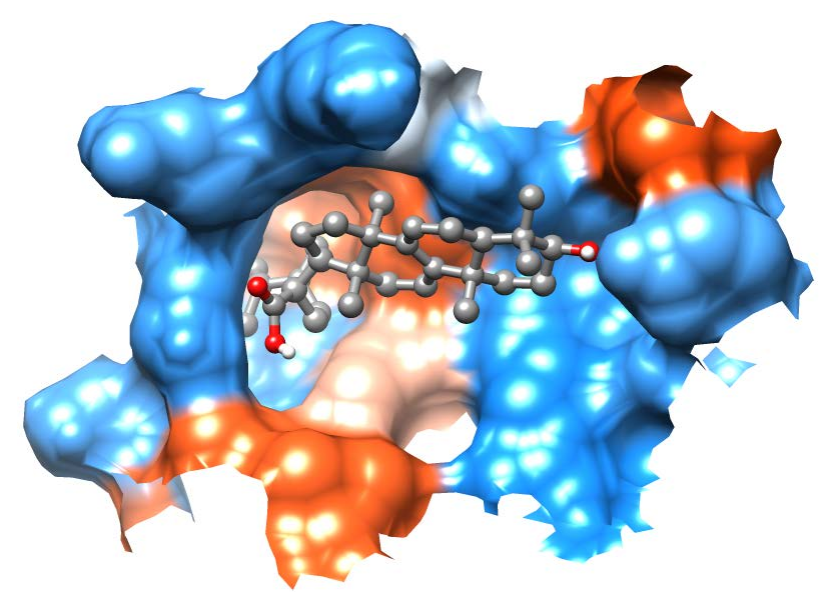

Figure 9. Hydrophobicity surface of amino acid residues within $5 \AA$ around DeEA.

\section{Conclusion}

The interaction between DeEA and HSA was systematically investigated by fluorescence spectroscopy, synchronous fluorescence spectroscopy, three-dimensional fluorescence spectroscopy and molecular docking approach. The fluorescence spectroscopy results suggested that DeEA quenched the intrinsic fluorescence of HSA through the combination of static and dynamic quenching mechanism. The thermodynamic parameters and molecular docking results indicated that the hydrogen bonding, hydrophobic force and van der Waals force were major acting forces during the binding process. DeEA could bind to HSA at $288 \mathrm{~K}$. However, DeEA could not be stored and carried by HSA in the body at higher temperature. The site marker competitive experiments and synchronous fluorescence spectroscopy revealed that the site II was the major binding site. According to the fluorescence resonance energy transfer theory, the energy transfer could occur with high probability and the binding distance was $3.29 \mathrm{~nm}$. The synchronous fluorescence spectroscopy and three-dimensional fluorescence spectroscopy results demonstrated that the binding process slightly changed conformation and microenvironment of HSA. The hydroxyl groups of DeEA were important groups during the binding process. The Glu-492 and Lys-545 were important amino acid residues for the interaction between DeEA and HSA. The interaction between DeEA and HSA was not characterized so far. Therefore, it was of vital significance to study on the interaction between DeEA and HSA. These experimental results will provide theoretical support for understanding the pharmacodynamics and pharmacokinetics properties of DeEA.

\section{Acknowledgements}

This work was supported by the National Natural Science Foundation of China (31270088); the program of introducing talents to universities on "creation and utilization of germplasm of mushroom crops" discipline innovation initiative (No. D17014) and the major project for agricultural application technology in- 
novation of Shandong province (2017). Thanks to Mr. Xiangming Chen, Binzhou Medical University, for assisting the fluorescence spectroscopy studies.

\section{Conflicts of Interest}

The authors declare no conflicts of interest regarding the publication of this paper.

\section{References}

[1] Gascoigne, R.M., Robertson, A. and Simes, J.J.H. (1953) The Chemistry of Fungi. Part XVII. Dehydroeburicoic Acid. Journal of the Chemical Society, 6, 1830-1837. https://doi.org/10.1039/jr9530001830

[2] Deng, J., Chen, S., Jow, G., Hsueh, C. and Jeng, C. (2009) Dehydroeburicoic Acid Induces Calcium- and Calpain-Dependent Necrosis in Human U87MG Glioblastomas. Chemical Research in Toxicology, 22, 1817-1826. https://doi.org/10.1021/tx9002275

[3] Guan-Jhong, H., Jeng-Shyan, D., Shyh-Shyun, H., Chao-Ying, L., Wen-Chi, H., Sheng-Yang, W., Ping-Jyun, S. and Yueh-Hsiung, K. (2013) Hepatoprotective Effects of Eburicoic Acid and Dehydroeburicoic Acid from Antrodia camphorata in a Mouse Model of Acute Hepatic Injury. Food Chemistry, 141, 3020-3027. https://doi.org/10.1016/j.foodchem.2013.03.061

[4] Deng, J.S., Huang, S.S., Lin, T.H., Lee, M.M., Kuo, C.C., Sung, P.J., Hou, W.C., Huang, G.J. and Kuo, Y.H. (2013) Analgesic and Anti-Inflammatory Bioactivities of Eburicoic Acid and Dehydroeburicoic Acid Isolated from Antrodia camphorata on the Inflammatory Mediator Expression in Mice. Journal of Agricultural and Food Chemistry, 61, 5064-5071. https://doi.org/10.1021/jf303820k

[5] Du, Y.C., Chang, F.R., Wu, T.Y., Hsu, Y.M., El-Shazly, M., Chen, C.F., Sung, P.J., Lin, Y.Y., Lin, Y.H., Wu, Y.C. and Lu, M.C. (2012) Antileukemia Component, Dehydroeburicoic Acid from Antrodia camphorata Induces DNA Damage and Apoptosis in Vitro and in Vivo Models. Phytomedicine, 19, 788-796.

https://doi.org/10.1016/j.phymed.2012.03.014

[6] Kuo, Y.H., Lin, C.H. and Shih, C.C. (2016) Dehydroeburicoic Acid from Antrodia camphorata Prevents the Diabetic and Dyslipidemic State via Modulation of Glucose Transporter 4, Peroxisome Proliferator-Activated Receptor Alpha Expression and AMP-Activated Protein Kinase Phosphorylation in High-Fat-Fed Mice. International Journal of Molecular Sciences, 17, 872. https://doi.org/10.3390/ijms17060872

[7] Kuo, Y.H., Lin, C.H. and Shih, C.C. (2015) Antidiabetic and Antihyperlipidemic Properties of a Triterpenoid Compound, Dehydroeburicoic Acid, from Antrodia camphorata in Vitro and in Streptozotocin-Induced Mice. Journal of Agricultural and Food Chemistry, 63, 10140-10151. https://doi.org/10.1021/acs.jafc.5b04400

[8] Azzazy, H.M.E. and Christenson, R.H. (1997) All about Albumin: Biochemistry, Genetics, and Medical Applications. American Association for Clinical Chemistry Inc., 432-450.

[9] Shaw, A.K. and Pal, S.K. (2008) Resonance Energy Transfer and Ligand Binding Studies on pH-Induced Folded States of Human Serum Albumin. Journal of Photochemistry and Photobiology B: Biology, 90, 187-197. https://doi.org/10.1016/j.jphotobiol.2008.01.001

[10] Sugio, S., Kashima, A., Mochizuki, S., Noda, M. and Kobayashi, K. (1999) Crystal 
Structure of Human Serum Albumin at 2.5 Angstrom Resolution. Protein Engineering, Design and Selection, 12, 439-446. https://doi.org/10.1093/protein/12.6.439

[11] Sudlow, G., Birkett, D.J. and Wade, D.N. (1975) Characterization of 2 Specific Drug Binding-Sites on Human Serum Albumin. Molecular Pharmacology, 11, 824-832.

[12] Sudlow, G., Birkett, D.J. and Wade, D.N. (1976) Further Characterization of Specific Drug Binding Sites on Human Serum Albumin. Molecular Pharmacology, 12, 1052-1061.

[13] Zhang, G., Wang, L. and Pan, J. (2012) Probing the Binding of the Flavonoid Diosmetin to Human Serum Albumin by Multispectroscopic Techniques. Journal of Agricultural and Food Chemistry, 60, 2721-2729. https://doi.org/10.1021/jf205260g

[14] Yue, Y., Liu, J., Liu, R., Sun, Y., Li, X. and Fan, J. (2014) The Binding Affinity of Phthalate Plasticizers-Protein Revealed by Spectroscopic Techniques and Molecular Modeling. Food and Chemical Toxicology, 71, 244-253. https://doi.org/10.1016/j.fct.2014.06.022

[15] Yue, Y., Liu, J., Fan, J. and Yao, X. (2011) Binding Studies of Phloridzin with Human Serum Albumin and Its Effect on the Conformation of Protein. Journal of Pharmaceutical and Biomedical Analysis, 56, 336-342. https://doi.org/10.1016/j.jpba.2011.05.018

[16] Sjoholm, I., Ekman, B., Kober, A., Ljungstedtpahlman, I., Seiving, B. and Sjodin, T. (1979) Binding of Drugs to Human-Serum Albumin:XI. Specificity of 3 Binding-Sites as Studied with Albumin Immobilized Microparticles. Molecular Pharmacology, 16, 767-777.

[17] Park, H., Lee, J. and Lee, S. (2006) Critical Assessment of the Automated AutoDock as a New Docking Tool for Virtual Screening. Proteins. Structure Function and Bioinformatics, 65, 549-554. https://doi.org/10.1002/prot.21183

[18] Frisch, M.J. (2006) Optimizing Large Molecules with Gaussian 03. Chemicke Listy, 100, A9.

[19] Feroz, S.R., Mohamad, S.B., Bujang, N., Malek, S.N.A. and Tayyab, S. (2012) Multispectroscopic and Molecular Modeling Approach to Investigate the Interaction of Flavokawain B with Human Serum Albumin. Journal of Agricultural and Food Chemistry, 60, 5899-5908. https://doi.org/10.1021/jf301139h

[20] Goddard, T.D., Huang, C.C. and Ferrin, T.E. (2007) Visualizing Density Maps with UCSF Chimera. Journal of Structural Biology, 157, 281-287. https://doi.org/10.1016/j.jsb.2006.06.010

[21] Eftink, M.R. and Ghiron, C.A. (1981) Fluorescence Quenching Studies with Proteins. Analytical Biochemistry, 114, 199-227. https://doi.org/10.1016/0003-2697(81)90474-7

[22] Albrecht, C., Joseph, R. and Lakowicz, J.R. (2008) Principles of Fluorescence Spectroscopy, 3rd Edition. Analytical and Bioanalytical Chemistry, 390, 1223-1224. https://doi.org/10.1007/s00216-007-1822-x

[23] Lakowicz, J.R. and Weber, G. (1973) Quenching of Fluorescence by Oxygen-Probe for Structural Fluctuations in Macromolecules. Biochemistry, 12, 4161-4170. https://doi.org/10.1021/bi00745a020

[24] Kathiravan, A., Chandramohan, M., Renganathan, R. and Sekar, S. (2009) Spectroscopic Studies on the Interaction between Phycocyanin and Bovine Serum Albumin. Journal of Molecular Structure, 919, 210-214. https://doi.org/10.1016/j.molstruc.2008.09.005

[25] Ware, W.R. (1962) Oxygen Quenching of Fluorescence in Solution: An Experimen- 
tal Study of Diffusion Process. Journal of Physical Chemistry, 66, 455-458. https://doi.org/10.1021/j100809a020

[26] Gao, H., Lei, L.D., Liu, J.Q., Kong, Q., Chen, X.G. and Hu, Z.D. (2004) The Study on the Interaction between Human Serum Albumin and a New Reagent with Antitumour Activity by Spectrophotometric Methods. Journal of Photochemistry and Photobiology A: Chemistry, 167, 213-221. https://doi.org/10.1016/j.jphotochem.2004.05.017

[27] Suryawanshi, V.D., Anbhule, P.V., Gore, A.H., Patil, S.R. and Kolekar, G.B. (2012) Spectroscopic Investigation on the Interaction of Pyrimidine Derivative, 2-Amino6-Hydroxy-4-(3,4-Dimethoxyphenyl)-Pyrimidine-5-Carbonitrile with Human Serum Albumin: Mechanistic and Conformational Study. Industrial \& Engineering Chemistry Research, 51, 95-102. https://doi.org/10.1021/ie202005c

[28] Makarska-Bialokoz, M. (2018) Comparative Study of Binding Interactions between Porphyrin Systems and Aromatic Compounds of Biological Importance by Multiple Spectroscopic Techniques: A Review. Spectrochimica Acta Part A: Molecular \& Biomolecular Spectroscopy, 200, 263-274. https://doi.org/10.1016/j.saa.2018.04.037

[29] Makarskabialokoz, M. (2017) Interactions of Hemin with Bovine Serum Albumin and Human Hemoglobin: A Fluorescence Quenching Study. Spectrochimica Acta Part A: Molecular \& Biomolecular Spectroscopy, 193, 23-32. https://doi.org/10.1016/j.saa.2017.11.063

[30] Hu, Y.-J., Ou-Yang, Y., Dai, C.-M., Liu, Y. and Xiao, X.-H. (2010) Site-Selective Binding of Human Serum Albumin by Palmatine: Spectroscopic Approach. Biomacromolecules, 11, 106-112. https://doi.org/10.1021/bm900961e

[31] Ross, P.D. and Subramanian, S. (1981) Thermodynamics of Protein Association Reactions: Forces Contributing to Stability. Biochemistry, 20, 3096-3102. https://doi.org/10.1021/bi00514a017

[32] Olsson, T.S.G., Williams, M.A., Pitt, W.R. and Ladbury, J.E. (2008) The Thermodynamics of Protein-Ligand Interaction and Solvation: Insights for Ligand Design. Journal of Molecular Biology, 384, 1002-1017. https://doi.org/10.1016/j.jmb.2008.09.073

[33] Tian, F.-F., Jiang, F.-L., Han, X.-L., Xiang, C., Ge, Y.-S., Li, J.-H., Zhang, Y., Li, R., Ding, X.-L. and Liu, Y. (2010) Synthesis of a Novel Hydrazone Derivative and Biophysical Studies of Its Interactions with Bovine Serum Albumin by Spectroscopic, Electrochemical, and Molecular Docking Methods. The Journal of Physical Chemistry $B, 114,14842-14853$. https://doi.org/10.1021/jp105766n

[34] Ni, Y., Liu, Q. and Kokot, S. (2011) Spectrophotometric Study of the Interaction between Chlorotetracycline and Bovine Serum Albumin Using Eosin Y as Site Marker with the Aid of Chemometrics. Spectrochimica Acta Part A: Molecular \& Biomolecular Spectroscopy, 78, 443-448. https://doi.org/10.1016/j.saa.2010.11.007

[35] Qi, Z.-D., Zhou, B., Xiao, Q., Shi, C., Liu, Y. and Dai, J. (2008) Interaction of Rofecoxib with Human Serum Albumin: Determination of Binding Constants and the Binding Site by Spectroscopic Methods. Journal of Photochemistry and Photobiology A: Chemistry, 193, 81-88. https://doi.org/10.1016/j.jphotochem.2007.06.011

[36] Forster, T. (1959) 10th Spiers Memorial Lecture. Transfer Mechanisms of Electronic Excitation. Discussions of the Faraday Society, 27, 7-17.

https://doi.org/10.1039/DF9592700007

[37] Yue, Y., Chen, X., Qin, J. and Yao, X. (2009) Spectroscopic Investigation on the Binding of Antineoplastic Drug Oxaliplatin to Human Serum Albumin and Molecular Modeling. Colloids and Surfaces B: Biointerfaces, 69, 51-57. 
https://doi.org/10.1016/j.colsurfb.2008.10.016

[38] Schuphan, W. (1965) Biochemists Handbook. Qualitas Plantarum et Materiae Vegetabiles, 12, 218-230.

[39] Valeur, B. and Berberan-Santos, M.N. (2012) Molecular Fluorescence: Principles and Applications. 2nd Edition, Wiley-VCH, Weinheim. https://doi.org/10.1002/9783527650002

[40] Zhou, J., Wu, X., Gu, X., Zhou, L., Song, K., Wei, S., Feng, Y. and Shen, J. (2009) Spectroscopic Studies on the Interaction of Hypocrellin A and Hemoglobin. Spectrochimica Acta Part A: Molecular \& Biomolecular Spectroscopy, 72, 151-155. https://doi.org/10.1016/j.saa.2008.09.009

[41] Mandal, P. and Ganguly, T. (2009) Fluorescence Spectroscopic Characterization of the Interaction of Human Adult Hemoglobin and Two Isatins, 1-Methylisatin and 1-Phenylisatin: A Comparative Study. The Journal of Physical Chemistry B, 113, 14904-14913. https://doi.org/10.1021/jp9062115

[42] Zaroog, M.S. and Tayyab, S. (2012) Formation of Molten Globule-Like State during Acid Denaturation of Aspergillus Niger Glucoamylase. Process Biochemistry, 47, 775-784. https://doi.org/10.1016/j.procbio.2012.02.008

[43] Zhang, J., Zhuang, S., Tong, C. and Liu, W. (2013) Probing the Molecular Interaction of Triazole Fungicides with Human Serum Albumin by Multispectroscopic Techniques and Molecular Modeling. Journal of Agricultural and Food Chemistry, 61, 7203-7211. https://doi.org/10.1021/jf401095n

[44] Wang, Y., Wang, X., Wang, J., Zhao, Y., He, W. and Guo, Z. (2011) Noncovalent Interactions between a Trinuclear Monofunctional Platinum Complex and Human Serum Albumin. Inorganic Chemistry, 50, 12661-12668.

https://doi.org/10.1021/ic201712e 